\title{
Comparative proteomic analysis of membranous nephropathy biopsy tissues using quantitative proteomics
}

\author{
WEIGUO SUI $^{1 *}$, RUOHAN ZHANG $^{1 *}$, JIEJING CHEN ${ }^{1}$, HUIYAN HE $^{1}$, ZHENZHEN CUI $^{1,2}$, \\ MINGLIN OU ${ }^{1}, \mathrm{LI} \mathrm{GUO}^{1}, \mathrm{SHAN} \mathrm{CONG}^{1}, \mathrm{WEN} \mathrm{XUE}^{1}$ and YONG DAI ${ }^{1,3}$ \\ ${ }^{1}$ Nephrology Department, Guilin $181^{\text {st }}$ Hospital, Guangxi Key Laboratory of Metabolic Disease Research, Guilin, \\ Guangxi 541002; ${ }^{2}$ College of Life Science, Guangxi Normal University, Guilin, Guangxi 541004; \\ ${ }^{3}$ Clinical Medical Research Center, The Second Clinical Medical College of Jinan University \\ (Shenzhen People's Hospital), Shenzhen, Guangdong 518020, P.R. China
}

Received April 29, 2014; Accepted November 18, 2014

DOI: 10.3892/etm.2015.2197

\begin{abstract}
Membranous nephropathy (MN) is a common cause of nephrotic syndrome in adults and the second leading cause of end-stage renal disease due to primary glomerulonephritis. The aim of the present study was to identify potential biomarkers of $\mathrm{MN}$ and further characterize these proteins by Gene Ontology (GO) analysis. Isobaric tags for relative and absolute quantification were used to compare the protein levels in tissues from $\mathrm{MN}$ patients and healthy individuals, and the combined samples were subsequently separated by specialized communications exchange. Mass spectrometry data acquisition was conducted using a 4800 Plus MALDI TOF/TOF tandem mass spectrometry device, and the results were subjected to statistical analysis. A total of 1,903 proteins were identified, with 423 proteins exhibiting a difference of $>1.5$-fold compared with the control group. Of these, 202 proteins were upregulated, while 221 proteins were downregulated. In conclusion, GO enrichment analysis revealed that the differentially expressed proteins were primarily mapped to the following GO terms: 'Immune response', 'immune effector process', 'activation of immune response' and 'positive regulation of immune system process'. The affected proteins may be associated with the pathogenesis of MN; thus, may represent candidate MN biomarkers.
\end{abstract}

\section{Introduction}

In adults, nephrotic syndrome is commonly caused by membranous nephropathy (MN), which is the second leading

Correspondence to: Dr Yong Dai, Clinical Medical Research Center, The Second Clinical Medical College of Jinan University (Shenzhen People's Hospital), 1,017 Dongmen North Road, Shenzhen, Guangdong 518020, P.R. China

E-mail: daiyong2222@gmail.com

${ }^{*}$ Contributed equally

Key words: membranous nephropathy, proteome, isobaric tags for relative and absolute quantification, gene ontology analysis cause of end-stage renal disease (ESRD) due to primary glomerulonephritis (1). During the early stages of MN, IgG4 targets the M-type phospholipase $\mathrm{A}_{2}$ receptor. The IgG4 complex and/or other immune complexes are deposited on the subepithelial area of the basement membrane (2). A previous study estimated that one-third of MN patients develop ESRD or chronic renal failure and eventually require a renal transplantation (3). Although clinical manifestations, urinalysis, clinical chemistry tests and renal histopathology can be used to diagnose glomerular diseases, a renal biopsy provides a definitive diagnosis (4). Renal biopsy is an invasive procedure with the potential risk of serious complications, including hematoma, infection and arteriovenous fistula (5). Therefore, a safer and more effective diagnostic method is desirable. Biomarkers represent a potentially alternative method for diagnosis.

Proteomic technologies have enabled the identification of novel protein biomarkers $(6,7)$. Technologies used for the discovery of protein biomarkers for glomerular diseases include two-dimensional (2D) gel electrophoresis, 2D differential in-gel electrophoresis, surface-enhanced laser desorption ionization time-of-flight (TOF) mass spectrometry (MS) and capillary electrophoresis-MS (4). Quantitative proteomic methods are used in the identification and quantification of proteins expressed at a genome-wide level or in a complex mixture (8). Isobaric tags for relative and absolute quantification (iTRAQ) is a technique developed by the Applied Biosystems Incorporation (8). Reagents used for iTRAQ consist of a peptide reactive group, a reporter group used in the analysis and a molecular mass balance group. iTRAQ is used to label samples with up to eight independent isobaric tags, which correspond to eight unique reporter ions (mass-to-charge ratio, 113-121). Therefore, quantitative information is obtained following integration of the peak areas for the eight different samples $(8,9)$.

iTRAQ has been applied in the proteomic analysis of tissues from various diseases, including endometrial carcinoma (10), head and neck squamous cell carcinoma (11) and colorectal cancer (12). However, iTRAQ technology has been rarely used in the analysis of $\mathrm{MN}$ renal tissues. In the present study, iTRAQ was used to analyze the total protein content of 
renal tissues from patients with MN. The aim of the present study was to identify a safe, alternative, diagnostic method for $\mathrm{MN}$, whilst improving the understanding of the mechanisms underlying the pathogenesis of MN.

\section{Subjects and methods}

$M N$ and control groups. Renal tissue was collected from six MN patients between March and August 2011 at the Guilin 181 ${ }^{\text {st }}$ Hospital (Guilin, China; Table I). The patients were diagnosed with MN through a biopsy, had a creatinine clearance level of $\geq 30 \mathrm{ml} / \mathrm{min} / 1.73 \mathrm{~m}^{2}$ and suffered from persistent proteinuria of $>5 \mathrm{~g} / 24 \mathrm{~h}$, although maximal tolerated angiotensin II was blockaded for at least four months (13). A renal biopsy was performed for all the patients with $\mathrm{MN}$, and the results were examined by a certified pathologist in a blind analysis (the pathologist was unaware of the clinical and laboratory data). The control group consisted of only four individuals with normal kidney function and with no clinical evidence of $\mathrm{MN}$, since normal renal tissue is difficult to collect and has a short storage life (Table I). Fig. 1 shows light photomicrographs (magnification, 200x) of the renal tissues from the MN patients, stained using hematoxylin-eosin and visualized under a microscopse (Nikon Coolscope II; Nikon Corporation, Tokyo, Japan). This study was performed according to the guidelines set forth by the Guilin $181^{\text {st }}$ Hospital and abides by the Declaration of Helsinki on ethical principles for medical research involving human subjects. Written informed consent was obtained from all the subjects or their guardians.

Sample preparation. Biopsy samples were collected from the MN patients and control group. The samples were immediately washed with $0.9 \%$ RNase-free $\mathrm{NaCl}$ and briefly immersed in RNase inhibitor (Epicentre, Madison, WI, USA), according to the manufacturer's instructions. The samples were stored at $-80^{\circ} \mathrm{C}$ for subsequent analysis.

Protein extraction and quantification. Renal tissue samples (250 mg) collected from the MN patients and control group were ground into a fine powder in liquid nitrogen, and supplemented with acetone. Subsequently, 10\% trichloroacetic acid in acetone was added and the samples were incubated for $2 \mathrm{~h}$ at $-20^{\circ} \mathrm{C}$. Total protein was extracted using an extraction buffer, consisting of $8 \mathrm{M}$ urea, $4 \%$ CHAPS, $40 \mathrm{mM}$ Tris, $1 \mathrm{mM}$ phenylmethylsulfonyl fluoride, $2 \mathrm{mM}$ EDTA, $10 \mathrm{mM}$ dithiothreitol and $0.5-2 \%$ isotonic glucose phosphate buffer ( $\mathrm{pH}$ 8.5). Next, the samples were subjected to centrifugation at $40,000 \mathrm{x}$ g for $1 \mathrm{~h}$ at $10^{\circ} \mathrm{C}$. The protein concentration of the supernatant was determined using a bicinchoninic acid assay kit (Pierce Biotechnology, Inc., Rockford, IL, USA), according to the manufacturer's instructions.

iTRAQ reagent labeling, strong cation exchange (SCX) fractionation and tandem mass spectrometry (MS/MS). Total protein in each group was pooled, blocked, digested and labeled according to the iTRAQ protocol (Applied Biosystems Life Technologies, Foster City, CA, USA). The iTRAQ tags were as follows: Healthy control, iTRAQ 113; MN, iTRAQ 119. The labeled digests were subsequently combined into one sample mixture.
Table I. Main clinical and biochemical characteristics of patients with MN and the control group.

\begin{tabular}{lcc}
\hline Characteristic & MN patients & Control group \\
\hline Male/female, $\mathrm{n}$ & $5 / 1$ & $3 / 1$ \\
Age, years & $47.17 \pm 12.17$ & $39.52 \pm 17.23$ \\
Blood pressure, $\mathrm{mmHg}$ & $143 \pm 26 / 75 \pm 12$ & $122 \pm 12 / 73 \pm 11$ \\
Urinary protein & & \\
excretion, $\mathrm{g} / 24 \mathrm{~h}$ & $6.7 \pm 3.45$ & - \\
Serum creatinine, $\mathrm{mg} / \mathrm{dl}$ & $1.21 \pm 0.52$ & - \\
Creatinine clearance, $\mathrm{ml} / \mathrm{min}$ & $73.54 \pm 29.75$ & - \\
\hline
\end{tabular}

MN, membranous nephropathy. -, within normal range (Urinary protein excretion, $<0.15 \mathrm{~g} / 24 \mathrm{~h}$; serum creatinine, $0.15-1.2 \mathrm{mg} / \mathrm{dl}$; creatinine clearance, $95-102 \pm 20 \mathrm{ml} / \mathrm{min}$, where males had a value of $102 \pm 20 \mathrm{ml} / \mathrm{min}$ and females $95 \pm 20 \mathrm{ml} / \mathrm{min}$ ).

Multidimensional liquid chromatography was performed to separate the tryptic peptides prior to MS. The combined samples were separated into ten SCX fractions using a $3.5-\mu \mathrm{m}$ particle size column (35x0.3 mm, 300 ̊̊; Zorbax Bio-SCX Series II; Agilent Technologies, Inc., Santa Clara, CA, USA) with a potassium formate gradient in $25 \%$ acetonitrile. The peptide fractions were further separated on a Tempo ${ }^{\mathrm{TM}}$ liquid chromatography nanoflow and matrix-assisted laser desorption/ionization (MALDI) spotting system (Applied Biosystems), equipped with a reversed-phase Magic C18Aq column (Applied Biosystems). Each chromatography run yielded $\sim 380$ MALDI spots on a stainless steel MALDI target plate (Applied Biosystems) (14).

A 4800 Plus MALDI TOF/TOF ${ }^{\mathrm{TM}}$ analyzer (Applied Biosystems Life Technologies) was used for MS data acquisition. Signal-to-noise ratios of $\geq 40$ were required for MS/MS. Mass spectra from 500 laser shots were acquired for each spot. The MS/MS data from the ten SCX fractions were combined and analyzed using the Paragon Algorithm search engine and Human v3.62 (European Bioinformatics Institute, http://www.ebi.ac) (14).

Statistical and Gene Ontology (GO) analyses. Proteins yielding tryptic peptides with average reporter ion ratios of $\geq 1.5$ and $\leq 0.67$ were classified as upregulated and downregulated, respectively. The GO database annotates selected proteins according to their molecular function (MF), cellular component (CC) and biological process (BP). To investigate the functions of the identified proteins, the online tool, Web Gene Ontology Annotation Plotting (http://wego.genomics.org.cn/), was used.

\section{Results}

Proteome of renal tissue. Using a peptide of $>1$ and a confidence interval of $>95 \%(\mathrm{P}<0.05)$ as the cutoff values for protein identification, a total of 1,903 proteins were identified and quantified from the collected renal tissues. Of the 423 proteins with $>1$.5-fold differences, 202 proteins were upregulated, while 221 proteins were downregulated. The beta-2-microglobulin level of MN was 1.56 times higher compared with the control group. 

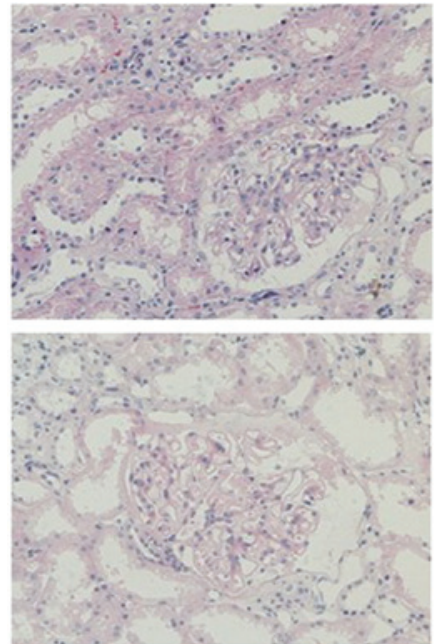
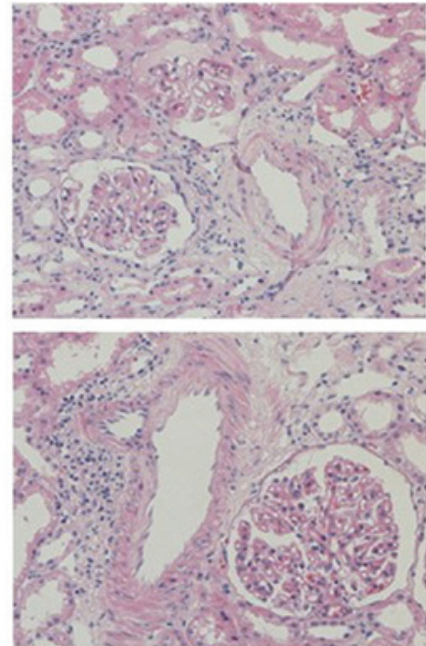
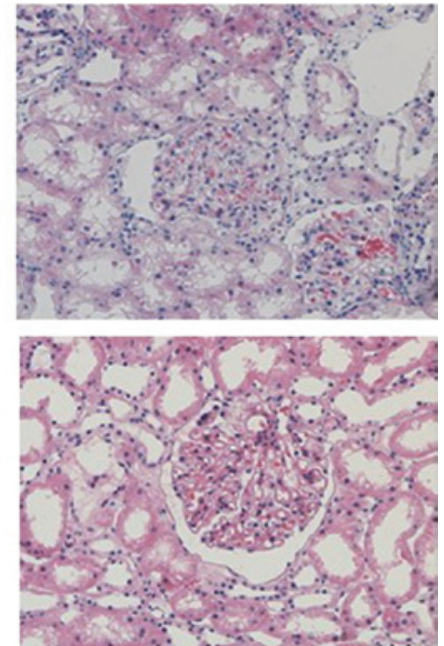

Figure 1. Images of renal tissue serial semi-thin sections from six patients with membranous nephropathy (hematoxylin-eosin; magnification, x200).

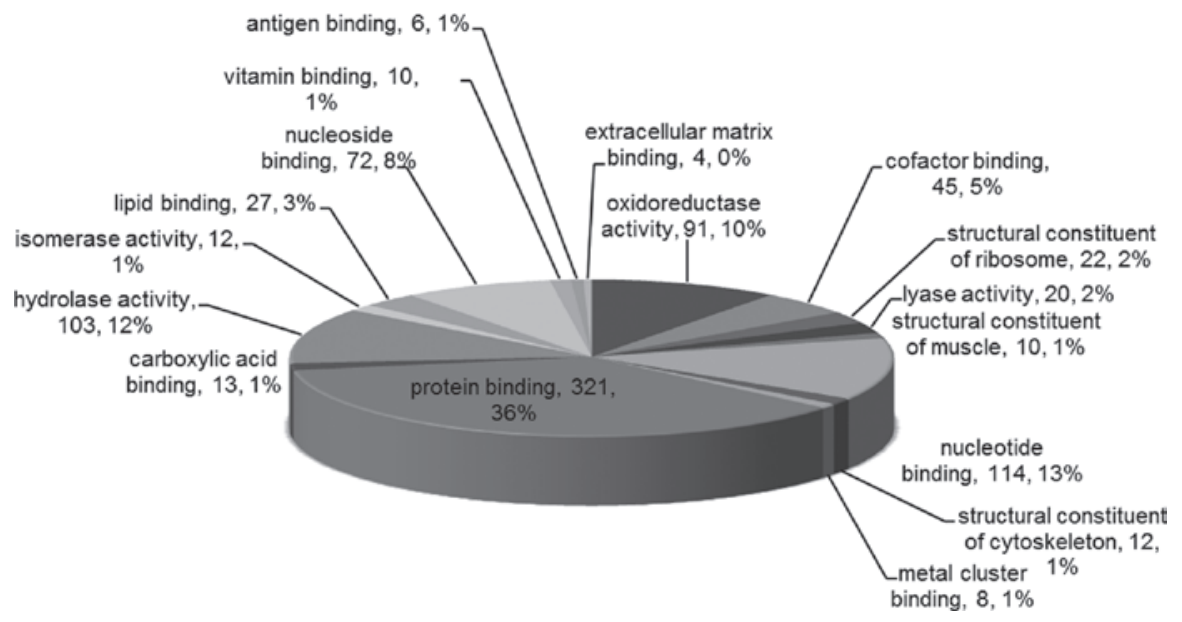

Figure 2. Enriched Gene Ontology molecular function terms for membranous nephropathy tissue proteins.

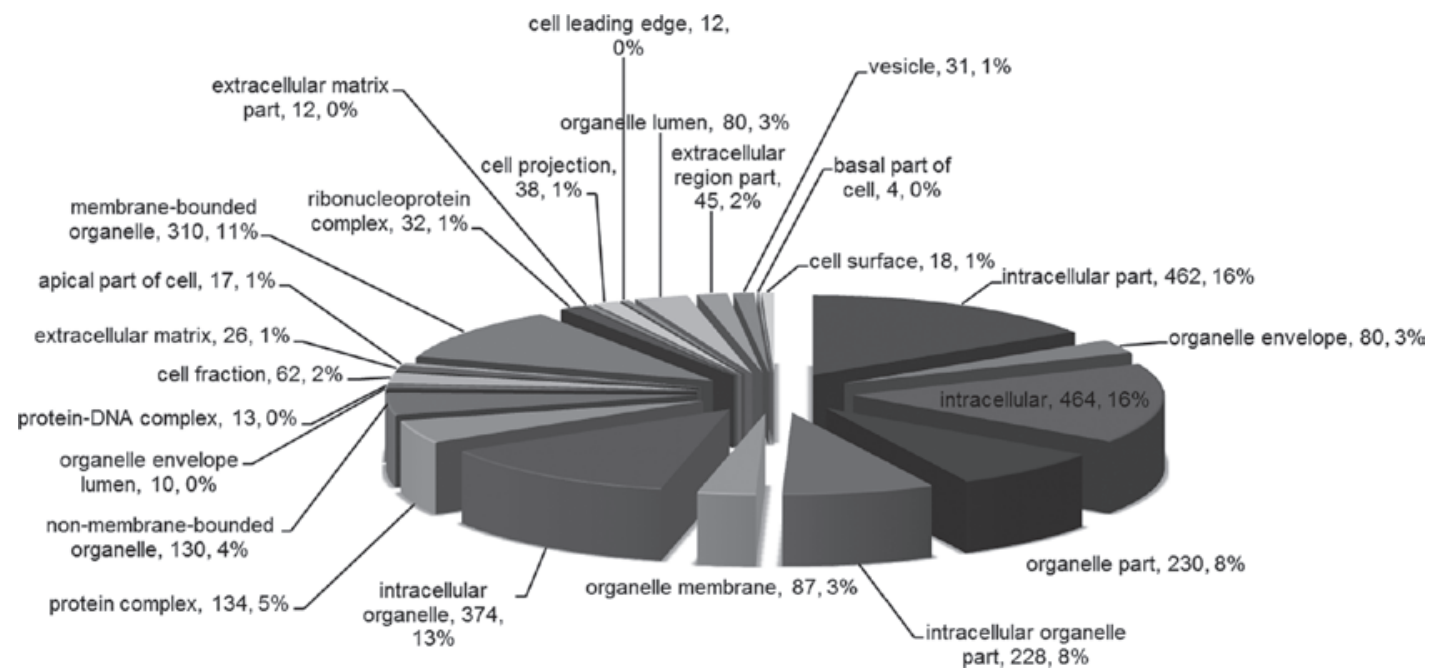

Figure 3. Enriched Gene Ontology cellular component terms for membranous nephropathy tissue proteins.

All the proteins were associated with the GO categories, MF, CC and BP. The most enriched MF terms included 'protein binding', 'nucleotide binding', 'hydrolase activity', 'oxidoreductase activity' and 'nucleoside binding' (Fig. 2). In addition, the most enriched $\mathrm{CC}$ terms included 'intracellular', 'intracellular part', 'intracellular organelle', 
Table II. Upregulated and downregulated proteins, belonging to the terms 'immune response', 'immune effector process', 'activation of immune response' and 'positive regulation of immune system process'.

Uniprot

accession no.
Protein name

(Organism species $=$ Homo sapiens)
Peptides iTRAQ

(95\% CI) $119: 113$

\section{Upregulated}

Q9NZP8

P07437

P04233

P31146

P61769

P04264

P52566

P01911

P61769

P13746

Q9TQE0

Complement C1r subcomponent-like protein (GN, C1RL; PE, 1; SV, 2)

Tubulin $\beta$ chain (GN, TUBB; PE, 1; SV, 2)

HLA class II histocompatibility antigen $\gamma$ chain (GN, CD74; PE, $1 ; \mathrm{SV}, 3)$

Coronin-1A (GN, CORO1A; PE, 1; SV, 4)

$\beta_{2}$-microglobulin (GN, B2M; PE, 1 ; SV, 1)

Keratin, type II cytoskeletal 1 (GN, KRT1; PE, 1; SV, 6)

Rho GDP-dissociation inhibitor 2 (GN, ARHGDIB; PE, 1; SV, 3)

HLA class II histocompatibility antigen, DRB1-15 $\beta$ chain (GN, HLA-DRB1; PE, 1; SV, 2)

$\beta_{2}$-microglobulin (GN, B2M; PE, 1; SV, 1)

HLA class I histocompatibility antigen, A-11 $\alpha$ chain (GN, HLA-A; PE, 1; SV, 1)

HLA class II histocompatibility antigen, DRB1-9 $\beta$ chain

(GN, HLA-DRB1; PE, 2; SV, 1)

P01857 Ig $\gamma$-1 chain $C$ region (GN, IGHG1; PE, 1; SV, 1)

P01594 Ig $\kappa$ chain V-I region AU (GN, KV102; PE, 1; SV, 1)

Q9NZ08 Endoplasmic reticulum aminopeptidase 1 (GN, ERAP1; PE, 1; SV, 3)

Q96A32

P32455

Myosin regulatory light chain 2, skeletal muscle isoform (GN, MYLPF; PE, 2; SV, 1)

P30481

P02794

Interferon-induced guanylate-binding protein 1 (GN, GBP1; PE, 1; SV, 1)

HLA class I histocompatibility antigen, B-44 $\alpha$ chain (GN, HLA-B; PE, 1; SV, 1)

P19320-2

Ferritin heavy chain (GN, FTH1; PE, 1; SV, 2)

Downregulated

P04003 C4b-binding protein $\alpha$ chain (GN, C4BPA; PE, 1; SV, 2)

P10809 $60 \mathrm{kDa}$ heat shock protein, mitochondrial (GN, HSPD1; PE, 1; SV, 2)

$\begin{array}{rl}1 & 2.3562 \\ 42 & 2.0528 \\ 1 & 2.0146 \\ 5 & 1.5730 \\ 4 & 1.5640 \\ 46 & 1.5123 \\ 5 & 1.6968 \\ 5 & 1.7591 \\ 4 & 1.5640 \\ 12 & 1.8167 \\ 7 & 1.6628 \\ & \\ 67 & 1.5854 \\ 4 & 1.6595 \\ 3 & 1.7790 \\ 4 & 2.9194 \\ 10 & 1.5951 \\ 4 & 2.5680 \\ 6 & 2.0462 \\ 3 & 1.6794\end{array}$

Q07021

Complement component $1 \mathrm{Q}$ subcomponent-binding protein, mitochondrial

(GN, C1QBP; PE, 1; SV, 1)

GN, gene name; PE, protein existence; SV, sequence version; MN, membranous nephropathy; HLA, human leukocyte antigen; CI, confidence interval.

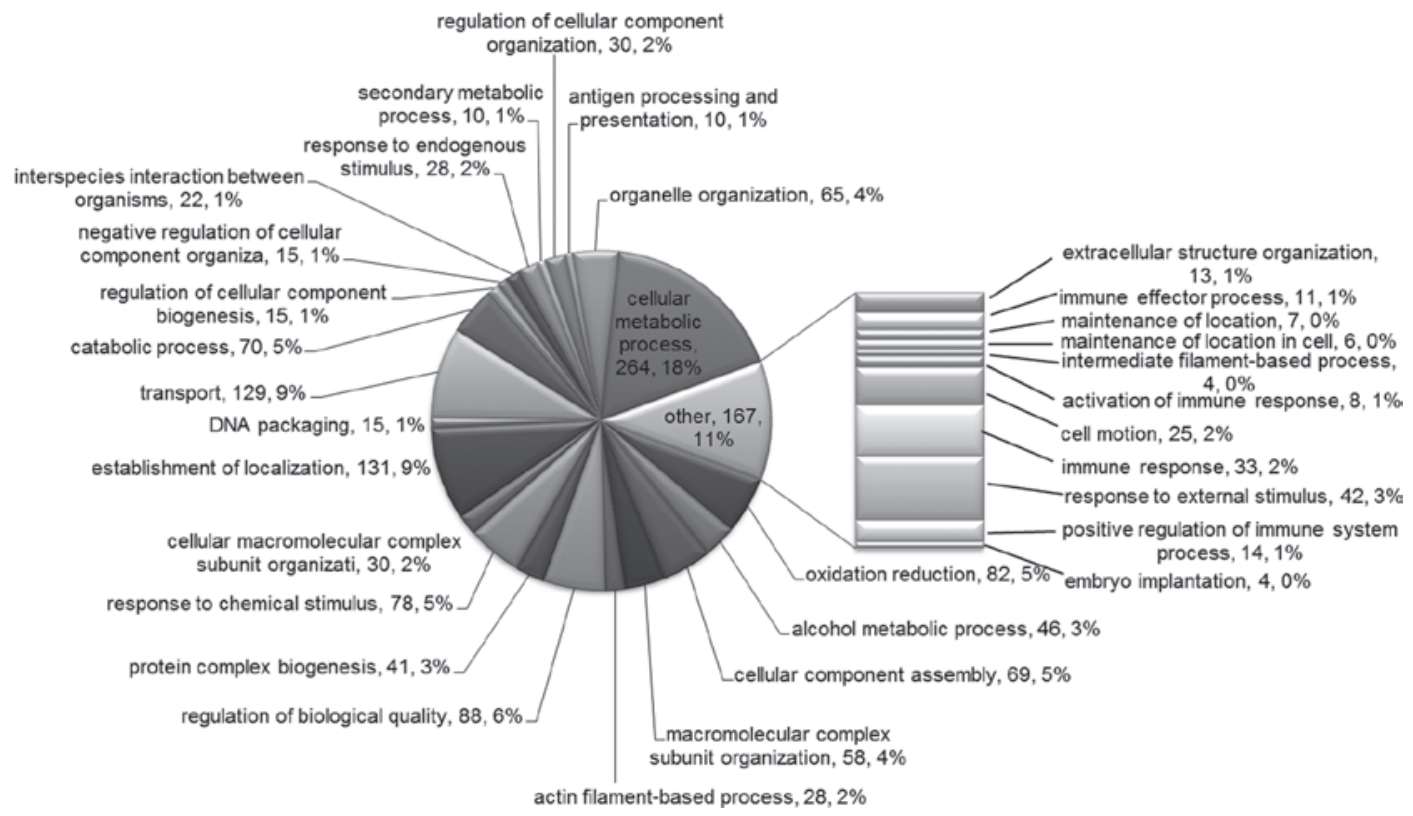

Figure 4. Enriched Gene Ontology biological process terms for membranous nephropathy tissue proteins. 
Table III. Proteins belonging to the specific biological process terms of the Gene Ontology enrichment analysis.

Term

Immune response

Immune effector process

Activation of immune response

Positive regulation of immune system process
Uniport accession no.
P01031, P07437, P01876, P40306, P04003, Q9NZP8, P52566, Q07021, P30499, P01911, P61769, P01834, P13746, P28062, Q9TQE0, P01857, P04264, P63104, P31146, P01594, P02788, Q9Y3Z3, Q9NZ08, Q96A32, P04433, P01903, P04233, P10809, P05156, P32455, P30481, P13796, P02794 P01903, P01031, P07437, P04233, P04003, P05156, P10809, Q9NZP8, Q9Y3Z3, P04264, P63104

P01031, P04003, P05156, P10809, Q9NZP8, P28482, P04264, P04216 P19320, P01031, P31146, P04003, Q9NZP8, P04216, P01903, P61769, $\mathrm{P} 04233, \mathrm{P} 05156, \mathrm{P} 10809, \mathrm{P} 28482, \mathrm{P} 04264, \mathrm{Q} 08722$ 'membrane-bounded organelle' and 'organelle part' (Fig. 3). Finally, the most enriched BP terms included 'cellular metabolic process', 'establishment of localization', 'transport', 'regulation of biological quality' and 'oxidation reduction' (Fig. 4).

The upregulated and downregulated proteins belonging to the terms 'immune response', 'immune effector process', 'activation of immune response' and 'positive regulation of immune system process' are shown in Tables II and III.

\section{Discussion}

The development of iTRAQ has enhanced the analysis of differential protein expression. Protein quantification using iTRAQ has been proposed as a suitable method for biomarker detection, since it permits parallel comparisons of protein abundance by measuring the peak intensities of reporter ions released from iTRAQ-tagged peptides. In the present study, iTRAQ technology and GO analysis were employed to perform quantitative proteomic analysis of plasma in $\mathrm{MN}$ tissue. A general proteome database was constructed for the renal tissue proteome, which has not been previously reported.

The use of GO proteomic analysis to investigate the observed changes was a necessary first step towards understanding the pathogenesis of MN. Differential proteins were assigned to the MF, CC and BP terms (Fig. 2-4), and GO enrichment analysis for the BP domain (Table III) revealed clusters of proteins for the following terms: 'Immune response', 'immune effector process', 'activation of immune response' and 'positive regulation of immune system process'. Therefore, cellular and humoral immune mechanisms may play a major role in the pathogenesis of MN. By contrast, the subsequent progression to renal failure appears to be determined primarily by cell-mediated immunity. T-helper 2 (Th2) cells secrete a number of cytokines, including interleukin-4, -5, -10 and -13, which trigger B-cell activation and immunoglobulin synthesis. A previous study revealed that a predominance of Th2 cells may exist in MN patients, as shown by the presence of $\mathrm{IgG}$, particularly IgG4 (15). This predominance complements deposits in the glomeruli and is a subclass of the type- 2 immune response (15). B cell epitope spreading is a process whereby the primary immune response against the dominant initiating epitope further extends to other epitopes, either within the same molecule or among different molecules. This phenomenon may be relevant to the pathogenesis of membranous disease (16). Mesangial cells may contribute to the derangements occurring in MN, which have features of immune effector cells (17).

Beyond the observed upregulation or downregulation of protein expression, the proteins listed in Table II provide evidence that specific proteins in the kidney tissue may play an important role in MN. These results may facilitate the analysis of the role of these proteins in MN and support the proteomic study of the kidney tissue. A number of the identified proteins were mapped to the GO terms, 'immune response', 'immune effector process', 'activation of immune response' and 'positive regulation of immune system process'. $\mathrm{MN}$ is considered to be an autoimmune disease, characterized by membrane-like thickening due to the accumulation of immune deposits on the outer glomerular basement membrane (16).

$\beta_{2}$-microglobulin is a highly accurate and specific prognosis predictor; therefore, this parameter should be evaluated to avoid unnecessary immunosuppressive therapy (18). A previous retrospective study indicated that the urinary levels of $\beta_{2}$-microglobulin and IgG are useful predictors of renal insufficiency in patients with MN (19). In the present study, $\beta_{2}$-microglobulin was found to be highly expressed in the kidney tissues of MN patients (1.56 times higher compared with the control group), and was associated with the GO terms, 'immune response' and 'positive regulation of immune system process'.

In conclusion, iTRAQ was used as a new strategy for proteomic analysis, and 1,903 proteins were found to be differentially expressed in the kidney tissues of MN patients when compared with the control group. GO enrichment analysis revealed that the differentially expressed proteins were primarily mapped to the GO terms, 'immune response', 'immune effector process', 'activation of immune response' and 'positive regulation of immune system process'. The identified proteins may be associated with the pathogenesis of MN; thus, may be candidate biomarkers for the disease. However, these proteins require further verification.

\section{Acknowledgements}

The authors thank the patients and healthy volunteers who participated in the study, and the members of staff for their assistance. This study was supported by the Planned Mission Statement about Construction Projects of Guangxi Science and Technology Infrastructure (Key Laboratory; no. 11-031-33), 
the Guangxi Key Laboratory Construction Project Planning Program (no. 12-071-32) and the Guangxi Natural Science Foundation of China (2012; no. GXNSFDA053017).

\section{References}

1. Papasotiriou M, Kalliakmani P, Huang L, et al: Does treatment with corticosteroids and cyclosporine reduce transglutaminase type 2 expression in the renal tissue of patients with membranous nephropathy? Nephron Clin Pract 121: c60-c67, 2012.

2. Beck LH Jr, Bonegio RG, Lambeau G, et al: M-type phospholipase A2 receptor as target antigen in idiopathic membranous nephropathy. N Engl J Med 361: 11-21, 2009.

3. Ngai HH, Sit WH, Jiang PP, et al: Serial changes in urinary proteome profile of membranous nephropathy: implications for pathophysiology and biomarker discovery. J Proteome Res 5: 3038-3047, 2006.

4. Thongboonkerd V: Biomarker discovery in glomerular diseases using urinary proteomics. Proteomics Clin Appl 2: 1413-1421, 2008.

5. Whittier WL and Korbet SM: Renal biopsy: update. Curr Opin Nephrol Hypertens 13: 661-665, 2004.

6 . Veenstra TD: Global and targeted quantitative proteomics for biomarker discovery. J Chromatogr B Analyt Technol Biomed Life Sci 847: 3-11, 2007.

7. Duncan MW and Hunsucker SW: Proteomics as a tool for clinically relevant biomarker discovery and validation. Exp Biol Med (Maywood) 230: 808-817, 2005.

8. Sun C, Song C, Ma Z, et al: Periostin identified as a potential biomarker of prostate cancer by iTRAQ-proteomics analysis of prostate biopsy. Proteome Sci 9: 22, 2011.

9. Sui W, Tang D, Zou G, et al: Differential proteomic analysis of renal tissue in lupus nephritis using iTRAQ reagent technology. Rheumatol Int 32: 3537-3543, 2012.

10. DeSouza L, Diehl G, Rodrigues MJ, et al: Search for cancer markers from endometrial tissues using differentially labeled tags iTRAQ and cICAT with multidimensional liquid chromatography and tandem mass spectrometry. J Proteome Res 4: 377-386, 2005
11. Ralhan R, Desouza LV, Matta A, et al: Discovery and verification of head-and-neck cancer biomarkers by differential protein expression analysis using iTR AQ labeling, multidimensional liquid chromatography, and tandem mass spectrometry. Mol Cell Proteomics 7:1162-1173, 2008.

12. Chen JS, Chen KT, Fan CW, et al: Comparison of membrane fraction proteomic profiles of normal and cancerous human colorectal tissues with gel-assisted digestion and iTRAQ labeling mass spectrometry. FEBS J 277: 3028-3038, 2010.

13. Irazabal MV, Eirin A, Lieske J, et al: Low- and high-molecular-weight urinary proteins as predictors of response to rituximab in patients with membranous nephropathy: a prospective study. Nephrol Dial Transplant 28: 137-146, 2013.

14. Wang L, Dai Y, Qi S, et al: Comparative proteome analysis of peripheral blood mononuclear cells in systemic lupus erythematosus with iTRAQ quantitative proteomics. Rheumatol Int 32: 585-593, 2012.

15. Hirayama K, Ebihara I, Yamamoto S, et al: Predominance of type-2 immune response in idiopathic membranous nephropathy. Cytoplasmic cytokine analysis. Nephron 91: 255-261, 2002

16. Ronco P and Debiec H: Antigen identification in membranous nephropathy moves toward targeted monitoring and new therapy. J Am Soc Nephrol 21: 564-569, 2010.

17. Werber HI, Emancipator SN, Tykocinski ML and Sedor JR: The interleukin 1 gene is expressed by rat glomerular mesangial cells and is augmented in immune complex glomerulonephritis. J Immunol 138: 3207-3212, 1987.

18. Hofstra JM, Deegens JK, Willems HL and Wetzels JF: Beta-2-microglobulinis superiorto $\mathrm{N}$-acetyl-beta-glucosaminidase in predicting prognosis in idiopathic membranous nephropathy. Nephrol Dial Transplant 23: 2546-2551, 2008.

19. Branten AJ, du Buf-Vereijken PW, Klasen IS, et al: Urinary excretion of beta2-microglobulin and IgG predict prognosis in idiopathic membranous nephropathy: a validation study. J Am Soc Nephrol 16: 169-174, 2005. 